\title{
SERBILUZ: Transitando hacia un modelo gerencial innovador
}

\author{
Fernández, Norcka* \\ Delgado, Francys ${ }^{* *}$ \\ Ortega, Egla ${ }^{\star \star *}$ \\ Caldera, Edixson ${ }^{\star \star \star \star}$
}

\section{Resumen}

El propósito de este trabajo fue explorar en el desarrollo del Sistema de Servicios Bibliotecarios y de Información de la Universidad del Zulia (SERBILUZ) los elementos fundamentales de la nueva gerencia pública (NGP) basados en el Modelo de las Cinco "R" de Osborne y Gaebler (1999). A tal efecto se revisaron documentos normativos y de gestión de la Universidad del Zulia (LUZ) y de SERBILUZ, conjuntamente con el análisis de enfoques conceptuales los cuales sirvieron de fundamentación teórica a esta investigación de tipo documental. Los resultados evidenciaron que el recurso humano necesita motivación, capacitación e incentivos laborales y profesionales, la info y tecnoestructura requiere de recursos institucionales, humanos y financieros, así como de alianzas estratégicas con instituciones afines en el ámbito nacional e internacional Se concluye que la estructura organizativa de SERBILUZ fue replanteada con base a las tendencias de la NGP establecidas en el Modelo de las Cinco "R" y con el Plan de Desarrollo Estratégico de LUZ.

Palabras clave: Modelo de las Cinco "R"; nueva gerencia pública; sistemas de información; gestión de la información.

\section{Recibido: 24-05-05. Aceptado: 22-11-06}

* Doctora en Ciencias Gerenciales, Docente Titular, dedicación exclusiva de la Escuela de Bibliotecología y Archivología Investigadora adscrita al Centro de Investigación de la Empresa. Miembro del Programa de Promoción de la Investigación (PPI).

E-mail: norkalibertad@gmail.com

** Doctora en Ciencias. Mención Investigación, Docente Titular a tiempo convencional de la Escuela de Bibliotecología y Archivología. LUZ. E-mail: fradel16@yahoo.com

*** Magíster en Bibliotecología y Ciencias de la Información. Docente Titular, dedicación exclusiva de la Escuela de Bibliotecología y Archivología. Investigadora adscrita al Centro de Estudios Históricos y al Centro de la Comunicación y la Información ( $\mathrm{ClCl})$. Miembro del Programa de Promoción de la Investigación (PPI).E-mail: eglaortega@gmail.com

**** Licenciado en Bibliotecología y Archivología. Docente Asistente, Tiempo Completo adscrito a la Escuela de Bibliotecología y Archivología. Director de SERBILUZ.E-mail: ejocal@yahoo.es 


\section{SERBILUZ: Moving Towards an Innovative Managerial Model}

\section{Abstract}

The objective of this research was to explore the development of the System of Library and Information Services of the University of Zulia (SERBILUZ), based on the fundamental elements of new public management based on the model of the five "R"s of Osborne and Gaebler (1999). To do this, normative management documents at the University of Zulia (LUZ) and SERBILUZ were reviewed, and an analysis of conceptual approaches was undertaken which served as a theoretical foundation for this documentary research. The results evidenced that human resources required motivation, training, labor and professional incentives, and the info and techno-structure requires institutional, human and financial resources, as well as strategic alliances with related institutions on a national and international plane. The conclusion is that the organizational structure of SERBILUZ was redesigned, based on NGB tendencies established in the model of the five "Rs" and with a Strategic Development Plan.

Key words: Model of the five "R", new public management, information system, information management.

\section{Introducción}

Frente a los retos que impone la sociedad actual, tales como: globalización, gestión comunicacional y desarrollo de nuevas tecnologías, el desempeño de la gerencia pública en el ámbito nacional y regional que involucra tanto a los entes gubernamentales como a los institucionales debería promover cambios direccionados a modificar la concepción habitual de conducir y dirigir la administración pública con base a esquemas que se amolden y respondan al concepto de una nueva gerencia pública (NGP) dinámica y progresista, tal como lo plantea Hodd (1994) quien la considera como un cambio en el diseño organizacional de la gestión pública basada en nuevos valores.

En este sentido es fundamental llevar la organización hacia la consecución de sus objetivos sustentados en la ética, talento humano y valores. La toma de de- cisiones estaría así orientada en concordancia con las necesidades de la organización a fin de evitar la lentitud en los procesos, la inercia en los mandos, la reiterada justificación para no ejercer o desarrollar los compromisos institucionales, la desorganización y la no información que impiden la funcionabilidad de la gestión pública.

La acción forma parte de la dinámica institucional, podemos decir, que es el motor de los procesos o la sinergia para lograr en el tiempo previsto y en el espacio correspondiente, los propósitos institucionales, así es necesario, centrarse en el ejercicio que impone el liderazgo el cual se concibe, como, "el arte de influir sobre la gente para que trabaje con entusiasmo en la consecución de objetivos en pro del bien común" (Hunter, 1999:38).

La Universidad del Zulia (LUZ) como institución pública, no está ajena a estos procesos de cambio. El reto de 
transformarla impone la realización de actividades que están dentro de los procesos administrativos, donde la planificación de las actividades es fundamental para poner en marcha el compromiso social, la generación de conocimiento, la excelencia académica, tal cual se menciona en su filosofía de gestión.

LUZ inició en el período correspondiente a la gestión 1996-2000, los procesos de transformación, apoyada en la resolución No. 336 del Consejo Universitario (CU) que incluye el plan de desarrollo estratégico donde se plantea la transformación de los niveles estratégico, táctico y operativo, para la conducción, dirección y racionalización de los procesos, que permitan viabilizar la estructura organizativa, haciéndola más productiva con sentido de pertinencia, compromiso social y excelencia académica.

Basado en esta resolución (No. 336), el Sistema de Servicios Bibliotecarios y de Información de la Universidad del Zulia (SERBILUZ), adscrito al ViceRectorado Académico con una gestión enmarcada en las tendencias auspiciadas por la nueva gerencia pública, responde a los desafíos que confronta el país en materia gerencial, informacional y de servicios, conjuntamente con las disposiciones de cambios y retos para la transformación contempladas por LUZ, el cual debe asumirse para la transformación que se requiere "como un proceso dinámico continuo que permite organizar de manera sistemática y con conocimiento de futuro, los programas y proyectos estratégicos" (Dirección General de Planificación Universitaria (1996-2000).

Este estudio tuvo como objetivo explorar en el desarrollo de SERBILUZ los elementos fundamentales de la nueva gerencia pública basados en el modelo de las Cinco "R" de Osborne y Gaebler (1994). A tal efecto, se revisaron documentos normativos y de gestión tales como: Plan de Desarrollo Estratégico 1996-2000, Gestión de la Información y el Conocimiento, Taller Operativo entre otros, que conjuntamente con documentos bibliográficos y hemerográficos sobre gerencia pública, sirvieron de fundamentación teórica a esta investigación de carácter documental.

\section{Gerencia pública emergente}

La nueva gerencia pública NGP surge como una necesidad de enfrentar los cambios frente al manejo de los dogmas que se observan en las instituciones estatales que son regidas por parámetros convencionales que impiden el desarrollo de los procesos y proyectos con celeridad, limitando el manejo de los servicios y el desempeño laboral en forma eficiente. Esto debido a los obstáculos que presentan, por un lado, una estructura organizacional burocratizada y por otra parte, los procesos administrativos sometidos mayormente "a la incertidumbre e inestabilidad que produce la inmovilidad gubernativa frente al cambio" (Medellin Torres, 1997:39), así mismo la nueva gerencia pública "busca ofrecer mecanismos más eficientes para la entrega de bienes y servicios y al mismo tiempo incrementar los niveles de desempeño" Pérez Salgado (2000:84).

Frente al desafío que involucra la NGP con relación al modelo tradicional caracterizado según Méndez (1999) por la burocracia institucional, deficiencia comunicacional y verticalidad en las estructuras 
organizativas entre otras, se observa la disposición de generar cambios que contribuyan a flexibilizar tanto las estructuras como los procesos administrativos. Al respecto Shepered (1999) aborda la idea de que en la actualidad existe un gran esfuerzo en la mayoría de los países latinoamericanos en adoptar la NGP, ante el agotamiento del modelo burocrático y dada la necesidad de poner en práctica una gama de opciones, experimentos y nuevas ideas como el centro de atención en el mejoramiento de los resultados.

Algunas de las más importantes características que sugiere el autor sobre este nuevo enfoque son la delegación en la toma de decisiones y la orientación hacia: los resultados, los clientes y el mercado; todo lo cual redundará en una guía más estratégica para la formulación de políticas, modificación de las estructuras de los organismos, un sistema de administración financiera con hincapié en los productos y un sistema de personal con énfasis en el premio al desempeño.

Por su parte Pérez Salgado (2000) señala que la NGP tiene sus fundamentos en los principios de "Rational Choice", "Public Choice" y "Calidad Total" que integran la administración de negocios con la administración pública con el fin de lograr un sector público más eficiente, eficaz y responsable en la entrega de bienes y servicios; por lo tanto según el referido autor, son características de la NGP la reducción del tamaño de la administración pública y su descentralización, la búsqueda de la excelencia y la orientación hacia el servicio público.

Esto desde luego, involucra esfuerzos, competencias, liderazgo, participación, objetividad y equilibrio en el manejo operativo de todas las funciones, que caractericen un nuevo enfoque en materia de gerencia pública y que inducen a la búsqueda de la excelencia en todas las áreas que maneje la institución y sobre todo, que sus acciones se vean cristalizadas inmediatamente, en la producción de servicios a entera satisfacción del usuario.

A nuestro juicio, ciertamente el enfoque de NGP concuerda con los planteamientos sobre una gerencia más participativa y en transición cuyos rasgos emergentes se reflejan en: flexibilidad, adaptabilidad, amplio control, pocos niveles de jerarquía vertical, comunicaciones fluidas, toma de decisiones descentralizadas y baja formalización.

Así mismo refiere Quintín (2003) que el nuevo modelo de gerencia pública se caracteriza por una serie de componentes operativos, como: indicadores de gestión, compromisos de resultados (CDR), evaluación del desempeño, incentivos, y la rendición de cuentas. Estos componentes de la transformación administrativa van a servir para medir el rendimiento de la acción gerencial. Siguiendo al autor se describen los cinco procesos básicos que sustentan la nueva gestión pública:

- Indicadores de gestión: constituyen la expresión cuantitativa de los resultados más significativos de un determinado programa, proyecto o servicio publico.

- Compromisos de resultados (CDR): componente referido a los contratos que se celebran entre los organismos estatales que tienen en su haber la asignación de recursos y la formulación de políticas públicas. 
- Evaluación del desempeño: toda organización requiere del análisis de sus objetivos y metas propuestas en cada de sus funciones con el propósito de evaluar su desempeño a nivel de personal, mercado y servicios principalmente. La evaluación eficaz del desempeño, "debe reconocer el legitimo deseo de los empleados de progresar en el ejercicio de su profesión. Uno de los medios para la integración de las demandas de la organización con las necesidades individuales es la administración del desarrollo profesional..." (Koontz y Weihrich, 2004:406).

- Incentivos: es necesario que se cree y consolide una política de incentivos para estimular el desempeño eficiente del desarrollo laboral.

- Rendición de cuentas: puede hacerse por diferentes vías. Lo importante, es asumirlo como un compromiso de primer orden dentro del proceso de la gestión pública, donde a través de la aplicación de mecanismos transparentes se podrá dar fe de la utilización de los recursos y de como estos proporcionan un bienestar social.

Estos componentes operativos forman parte del proceso que se requiere para la transformación y cambios gerenciales. A través de ellos, se puede evaluar una gestión, analizar sus resultados, aplicar correctivos, determinar cuales fueron las actividades de mayor alcance y las de menor cumplimiento.

Por otra parte, Osborne y Gaebler (1999) proponen un modelo para la transformación de la gerencia pública basado en cinco conceptos conocidos como la cinco "R" de la nueva gerencia pública: Reestructuración, Reingeniería, Reinvención, Realineación Reconceptualización. Según lo señalan los autores y refrendado por Jones y Thompson (1999:2), la reestructuración conforma el primer paso para un proceso de cambio de la gerencia. A través de esta fase, se analizan primeramente, los elementos estructurales de la organización y se eliminarán todos aquellos que entorpezcan sus funciones y servicios. Al analizar las tareas se pueden redistribuir, o eliminar para hacer más viable la organización.

La reingeniería, constituye el segundo paso en el modelo de gerencia pública de Osborne y Gaebler (1999:6), quienes señalan que la "reingeniería se centra más en "empezar de nuevo" que en tratar de "arreglar" los problemas existente mediante soluciones parciales o temporales aplicando "paños calientes". La reingeniería exige pensar acerca de procesos y no acerca de funciones y posiciones establecidas en las jerarquías organizacionales", que busca la modernización y uso de la tecnología para agilizar tareas y funciones. Esto significa la necesidad de considerar los entorpecimientos y entrabamientos que presenta la organización en sus procesos y la forma como el recurso humano se desempeña dentro de ella.

La reingeniería se basa en: diseñar principios en función de un objetivo o resultado, en lugar de hacerlo en una sola función, hacer que quienes utilicen el producto de la actividad, la desarrollen y que los individuos procesen esa información, captar la información directamente de la fuente y coordinar actividades paralelas durante el desarrollo de las mismas, y no después que éstas se hayan realizado. 
El manejo de estos principios permite hacer un seguimiento a las funciones que se realizan en la organización. Si cada unidad se monitorea, el flujo de información, la detección de problemas, y el cumplimiento de las actividades será más efectivo, y las operaciones de la empresa confrontaran menos problemas.

Hammer y Champy (1997:34) coincidiendo con lo expuesto por Osborne y Gaebler, plantean que la "reingeniería es la revisión fundamental y el rediseño radical de los procesos para alcanzar mejoras espectaculares en medidas criticas y contemporáneas de rendimiento, tales como costos, calidad, servicio y rapidez".

En cualquier organización cuya finalidad operativa está centrada en servicios al cliente, bien sean de consumo masivo, o de otra índole, es importante la consideración de la reinvención, como tercer elemento en importancia, que según Osborne y Gaebler (1999:10), "requiere de pensamiento estratégico y planificación para desplazarse hacia el desarrollo de nuevos mercados y métodos de suministros y servicios".

Este es una de las características que requiere mayor atención, por cuanto toda institución gubernamental que trabaje en función del cliente, bien sea de consumo o de servicios, necesariamente debe tener por parte del organismo que lo respalda, mayor consideración y estímulo. El servicio debe establecerse como una prioridad en cualquier institución que lo imparta, y el cliente debe ser la principal prioridad para la organización.

La realineación, cuarto paso en el modelo de Osborne y Gaebler, se basa en cotejar la estructura de la organización con las estrategias desarrolladas para lo- grar las metas deseadas y para motivar a los gerentes y empleados, es decir promueve el cambio interior de la dependencia. Esta etapa del modelo requiere del "establecimiento de una estructura de responsabilidades en el seno de la organización" (Jones y Thompson, 1999:18).

El elemento final del modelo la reconceptualización hace énfasis en el pensamiento creativo básicamente en los mercados, los clientes, los productos y la organización del trabajo. La consideración de cada uno de ellos es vital para el desarrollo de la organización y el desempeño laboral. Impone a la organización la necesidad de crear, pensar, tomar decisiones y actuar velozmente.

Frente a las dificultades, las empresas deben hacer uso de todas las herramientas de aprendizaje. Uno de los elementos que rompen la cotidianidad y la crisis, es precisamente la creatividad. El modelo asume este criterio como desplazamiento de la tendencia general y tradicional de pensar y actuar en la organización, hacia el uso de nuevas maneras de autoaprendizaje. Si las organizaciones priorizarán este recurso natural del ser humano, los propósitos que le asisten a la organización en sus planes y programas, se manejarían con mayor celeridad. Como refieren Jones y Thompson (1999:22), "pensar creativamente acerca de los problemas difíciles..." es la expresión clave para manejar la gerencia pública.

Estos conceptos tratados por Osborne y Gaebler (1999) e integrados en un nuevo modelo de gerencia pública, pueden ser considerados por las organizaciones de manera total o parcial. Ello depende de la forma como cada una esté integrada y predispuesta a los procesos de cambios. 


\section{SERBILUZ: Razón de ser}

Respecto a la organización bibliotecaria en el ámbito de las universidades, según Regos Varela (1999) se distinguen fundamentalmente dos alternativas: el de biblioteca única centralizada utilizado en universidades de reciente creación y un sistema bibliotecario único funcionalmente coordinado pero orgánica y estructuralmente descentralizado en diferentes puntos de servicio que van desde las Bibliotecas de Facultades (BF) que ofrecen servicios a varias escuelas adscritas a una misma facultad, los Centros de Información y Documentación (CID) orientados a la atención especializada en un área especifica y una Biblioteca Central (BC) en la que suele residir la dirección del sistema, las jefaturas de las secciones y servicios centralizados.

El Sistema de Servicios Bibliotecarios y de Información de la Universidad del Zulia (SERBILUZ) responde al segundo modelo, el mismo esta integrado por 25 unidades de información que funcionan en las diferentes facultades y dependencias con el propósito de posibilitar la asimilación, transformación, generación y transferencia de conocimientos. Es un ente administrativo adscrito al Vice-Rectorado Académico de la Universidad del Zulia, cuyo núcleo coordinador es la Biblioteca Central Gral. Rafael Urdaneta. Dentro de sus principales objetivos se identifican los siguientes:

- Consolidar servicios y productos informacionales sustentados en las tecnologías de información (TIC), que respondan a los intereses de la docencia, investigación y extensión de la Universidad del Zulia.
- Liderar políticas orientadas al desarrollo de colecciones -en cualquier tipología documental- que respondan a los intereses de la comunidad universitaria.

- Consolidar e innovar permanentemente los servicios de apoyo en las estructuras universitarias vinculadas al desarrollo de la investigación y el postgrado.

- Estimular la educación continua y la especialización de los recursos humanos del sistema en la gerencia de la información y el conocimiento

- Desarrollar estrategias que propicien la incorporación de las unidades del sistema a las redes académicas intra y extra institucionales.

Su razón de ser se sustenta en la innovación y excelencia en la conceptualización y desarrollo de productos y servicios orientados a apoyar las actividades de docencia, investigación y extensión de la comunidad universitaria. Esto se enmarca dentro del concepto de desarrollo sostenido que según Álvarez (2004:6) implica "tener en cuenta algunos límites, propios de los recursos del medio ambiente, así como los conocimientos tecnológicos y la interrelación con los seres humanos".

El Reglamento General del Sistema de Servicios Bibliotecarios y de Información, aprobado por el Consejo Universitario en septiembre de 1983, constituye su base jurídico-legal; el mismo esta enmarcado en la normativa universitaria de la cual asume valores y políticas para establecer su identidad propia dentro del Alma Mater.

Como gestor de la información, SERBILUZ, adquiere, almacena, organi- 
za, sistematiza, difunde y divulga documentos e información requerida por estudiantes, profesores e investigadores a quienes corresponde cumplir los planes, programas y proyectos establecidos o propuestos para la docencia, investigación y extensión, prioritariamente en LUZ, pero sin olvidar el compromiso con otras instituciones de la región, el país y el mundo. Como ente responsable de ejecutar los programas y llevar a cabo las políticas informacionales en apoyo a la generación de información y conocimiento.

\section{Aplicación del modelo de las Cinco "R” en SERBILUZ}

Las transformaciones en la administración pública, no devienen de este siglo XXI, sino que corresponden a la década de los 80 del siglo XX, cuando la gestión pública comenzó a experimentar innovaciones importantes en el estilo de la gerencia tradicional y que hoy en día, se han mantenido como una manera de tratar la complejidad que se observa en la administración pública, por cuanto ésta requiere como afirma Ávalos Aguilar (2002:5) "de una redefinición de su estructura como funcionamiento para hacer frente a los retos...". No obstante, cada organización debe cumplir su misión de planificar, organizar, dirigir y controlar, ser competitiva, proactiva y productiva. De esta forma, se consagraría en el manejo de sus funciones. Su razón de ser como ente organizacional integral, puede justificarse en el tiempo, en los costos que genera, tanto en mantenimiento de la infraestructura, como de personal. Así mismo cumplir con su filosofía de gestión y su proceso de transformación.
Las cinco características señaladas en el modelo de gerencia pública, viene a conformar la primera fase de cambio en SERBILUZ, basado en el modelo de las cinco " $R$ " de Osborne y Gaebler (1999). En el Diagrama 1 se pueden apreciar los componentes del modelo. Cada uno de los elementos que lo integran, deben desarrollarse y atenderse en forma simultánea, para que cada etapa pueda ser exitosa.

La política de reestructuración y transformación de su estructura y procesos, se ha iniciado bajo las premisas de este modelo, por considerar que el mismo, primero está inmerso en los criterios de la administración pública y segundo, por cuanto no excluye en su orientación al factor humano como promotor y ejecutor de todas las operaciones que se realizan en la institución. En SERBILUZ, este modelo se propone como un cambio en el modo de gerencia tradicional de la institución, adecuándolo a las transformaciones que se han puesto de manifiesto en el Plan de Desarrollo Estratégico de LUZ correspondiente al período 1996-2000.

En su afán de responder a la reforma académico-administrativa, SERBILUZ se incluye en este plan, sumándose a todas las dependencias universitarias para la transformación y modernización de las estructuras organizacionales. A tal fin, la Comisión Delegada del C.U. designa en junio de 2003 una Comisión Reestructuradora* que acepta el reto del cambio, concentrando sus funciones básicamente en cuatro categorías: organización administrativa, inversión financiera, infoestructura y tecnoestructura. En cada una de estas categorías se utilizaron los indicadores de gestión para medir el cum- 


\section{Diagrama 1 SERBILUZ: Aplicación del modelo de las Cinco “R”.}

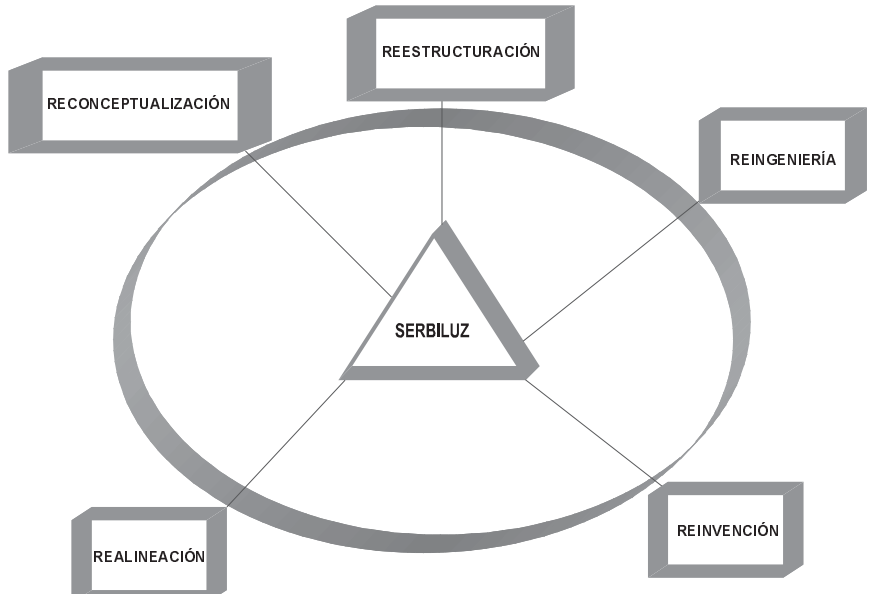

Elaborado con datos tomados del modelo de las cinco "R" de Osborne y Gaebler (1999). Adaptación Delgado y otros (2004).

plimiento de los objetivos, metas propuestas y determinar, a través de ello, el comportamiento y productividad institucional.

El modelo de gestión pública propuesto para SERBILUZ está sustentado en el modelo de las Cinco "R": reestructuración, reingeniería, reinvención, realineación y reconceptualización. Este modelo propone la optimización de los procesos administrativos y técnicos; garantizar la capacitación del personal del sistema tanto en lo técnico-administrativo, como en las áreas que contribuyan a la creación de un nuevo perfil del mismo; el fortalecimiento de las colecciones en formato digital; optimizar los procesos para la recuperación, análisis, conservación y difusión de la producción intelectual de LUZ, y de la memoria histórica del Zulia.

En el caso de SERBILUZ, se tomó en consideración la reestructuración or- ganizacional y funcional, de acuerdo a la categoría organización administrativa, la cual comprende los siguientes indicadores: 1) transformación organizacional, 2) proyectos de innovación y desarrollo, 3) evaluación y reactivación de cargos vacantes, 4) adiestramiento de personal, 5) divulgación y promoción de proyectos.

En relación con el indicador transformación organizacional, las propuestas estuvieron dirigidas en las políticas orientadas al desarrollo de colecciones; la vinculación con el Sector Socioproductivo regional y nacional; al programa de adiestramiento de personal; al sistema de desarrollo de Indicadores para SERBILUZ; a la redistribución de espacio físico y señalización; al redimensionamiento del proyecto SABIL y al análisis y evaluación de cargos vacantes en el sistema.

Los proyectos de innovación, contemplados en el proceso de transforma- 
ción fueron orientados en las siguientes áreas: teleinformación, (ampliación de sala de Internet de la biblioteca Claudio Muskus; Proyecto SABIL; Biblioteca PostGrado FHE-LUZ); planta física, patrimonio documental, evaluación, y el desarrollo de colecciones en formato digital. Es importante destacar en este proceso de reestructuración, la colaboración de la Escuela de Bibliotecología y Archivología en el desarrollo de planes conjuntos y obtención de recursos. Asimismo, el financiamiento de la tecnoestructura por parte de FUNDADESARROLLO cuya inversión fue de BS. 370.000.000,00.

En referencia con el adiestramiento, la Comisión Reestructuradora de SERBILUZ, priorizó la formación del talento humano, incrementando en un $147 \%$-en relación con el cuatrienio 2000-2003-, y el número de funcionarios que han formado parte del plan de adiestramiento. Así mismo, se diseñó una base de datos con la finalidad de tener un registro actualizado del personal, donde se reflejó la capacitación impartida y la detección de necesidades, tanto técnicas como administrativas y crecimiento personal.

Uno de los factores que permite medir el modelo, tanto en la fase inicial de reestructuración como en las siguientes, son las áreas que presentan debilidades: ese es el caso del indicador divulgación y promoción de proyectos-productos, donde se evaluó la poca presencia de SERBILUZ en eventos nacionales, a pesar de contar con personal calificado para mantener una participación activa en ellos.

En el redimensionamiento de los procesos en SERBILUZ, se consideró necesario el rediseño de sus esquemas funcionales y organizacionales a objeto de procurar mejoras en cada una de las actividades que así lo requerían. En este replanteamiento, se ha incluido la categoría general "Inversión Financiera" por cuanto su objetivo central está afianzado en la optimización del gasto y lo que representa para una organización como SERBILUZ.

La reingeniería se llevó a efecto en el manejo de los procesos, sus entrabamientos y en el desarrollo de estrategias que permitieron la obtención de un presupuesto acorde con las exigencias de la info y tecnoestructura demandadas por los usuarios.

Para el año 2004 y considerando que el "Consejo Nacional de Universidades establece que el $1.5 \%$ del presupuesto de la universidad, debe ser asignado al desarrollo y fortalecimiento de su sistema de bibliotecas" (Ortega y Ferrer, 2004: 18 ), se asignó un presupuesto (Bs. 1.249.144.390) con un incremento del $300 \%$, en relación con el de los años 2000-2003. En este período, el $80 \%$ de los recursos financieros disponibles se invirtieron en la suscripción de publicaciones científicas y bases de datos a texto completo. Ante esta situación, la Comisión Reestructuradora de SERBILUZ, con la finalidad de equilibrar la inversión, instrumentó un sistema híbrido en la compra de publicaciones científicas. En el mismo, se contempló la inclusión de colecciones en formato impreso y digital. Modalidad que representó un ahorro altamente significativo para la universidad, estimado en un $56,28 \%$.

Esto permitió a SERBILUZ el desarrollo de una Infoestructura, que posibilitó la disponibilidad y accesibilidad documental, así como la generación de nue- 
vos conocimientos, la visibilidad internacional de la producción intelectual universitaria, la creación de bases de conocimiento y la colección zuliana (Ortega y Ferrer, 2004:20).

El manejo de la información forma parte del núcleo central de funcionabilidad de SERBILUZ. Su empeño cada día en estar a la par de la socialización y generación del conocimiento no tiene limites. En este sentido, el desarrollo de colecciones, la suscripción a publicaciones científicas y bases de datos, cuenta para el año 2004 con 499 publicaciones periódicas en formato impreso en diferentes áreas del conocimiento y 12.148 en formato digital. Las bases de datos que ofrece son las siguientes: Academia Search Premier (multidisciplinaria), Business Search Premier (Área de Ciencias Sociales), American Chemical Society (Área de química y afines), loP. Institute of Physics (Área de física y afines). De acuerdo a Fernández, Delgado, Ortega y Caldera (2004:7) se logró "un incremento de $1.900 \%$ en la disponibilidad de revistas científicas a la comunidad académica y de investigación con relación a los años anteriores".

Se destaca en SERBILUZ las estrategias dirigidas a lograr la visibilidad internacional de la producción intelectual de la Universidad del Zulia, entre las cuales cabe citar la creación del sitio ScIELO* en LUZ, auspiciado por la organiza- ción Panamericana de la Salud y el Ministerio de Ciencia y Tecnología. Asimismo, el proyecto CYBERTESIS, auspiciado por la UNESCO, cuyo propósito es la publicación de las tesis de postgrado existentes en LUZ en las redes del conocimiento, ampliando el campo de divulgación de la producción intelectual universitaria.

En cuanto a la tecnoestructura, es importante hacer mención a la sustitución y repotenciación de equipos de computación y la sustentación de los procesos en el sistema de gestión documental Documanager**.

Con base en la reinvención se estudiaron los procesos con la finalidad de puntualizar la forma de dinamizar las funciones que se realizan en cada dependencia que conforman la estructura organizativa de SERBILUZ y así, a partir de indicadores, establecer estrategias de mercado de los productos y servicios del sistema.

En este contexto, se puede hacer referencia al indicador "Generación y Desarrollo de Conocimiento Competitivo", establecido con el propósito fundamental de evaluar el aporte de SERBILUZ en la investigación y desarrollo de conocimiento competitivo.

Todo ello, en alineación con los planteamientos de UNESCO (1996) en el marco del evento: "Los nuevos escenarios universitarios ante el fin de siglo", donde se exhorta las escuelas de biblio-

* SciELO: Scientific Electronic Library Online. Biblioteca electrónica de revistas científicas. Incluye, las revistas científicas Iberoamericanas y venezolanas. Este proyecto de carácter regional esta siendo desarrollado en Venezuela por el Ministerio de Ciencia y tecnología.

** Documanager: Sistema de Gestión Documental desarrollado por MULTIMEDIUM, con el fin de atender las necesidades de gestión y operación de unidades de información que exigen eficiencia, organización y rapidez en el ingreso, almacenamiento, búsqueda y recuperación de información. Su estructura de datos se apoya en las características establecidas por CDS/ISIS. 
tecología y a las bibliotecas universitarias, estableciendo de estrategias orientadas al fortalecimiento de los procesos de integración Inter-universitaria con el sector socio-productivo, ya que éstas constituirán los pilares básicos para la construcción del futuro académico de América Latina y el Caribe.

Cabe destacar que SERBILUZ asume como prioridad el facilitar a la comunidad universitaria, el acceso a la información y el conocimiento disponible en diferentes redes en el ámbito nacional e internacional y en las bases de datos referenciales y a texto completo más relevantes en el ámbito mundial y para ello cuenta con una novedosa infraestructura tecnológica.

De igual manera contribuye a la divulgación del conocimiento generado en pro del proceso socio-histórico cultural del Zulia y la identidad zuliana. Este aspecto es muy significativo, por cuanto SERBILUZ, a través del Centro de Investigación, Información y Documentación Histórica del Zulia (CIDHIZ), podrá preservar y conservar en cualquier formato la producción de información que tenga como expresión la zulianidad.

Así mismo se logró la divulgación de las investigaciones generadas en la Universidad del Zulia en la redes de conocimientos nacionales e internacionales. Se alcanza así, la participación activa de la comunidad académica de LUZ en la construcción de la plataforma intelectual que garantice la consolidación y generación de nuevos conocimientos.

Toda organización se mueve a través del recurso humano. Estos constituyen la fortaleza de cada institución pública o privada. Al respecto, tanto la Univer- sidad del Zulia como SERBILUZ, están conscientes de ello, y por eso su empeño en realinear las condiciones necesarias que permitan al personal realizar sus actividades laborales, dentro de un ambiente adecuado a las funciones de trabajo, con políticas de reconocimiento y estimulo al personal. Por supuesto, teniendo como referente el nivel de exigencias que se observa en el plan operativo y en los indicadores de gestión. En tal sentido, la organización promueve la capacitación, reconocimiento y evaluación del profesional y técnico a través de los indicadores de desempeño y de reuniones de crecimiento colectivo.

Como última fase del modelo la reconceptualización busca la reorientación estructural y funcional de la organización, la observación, la decisión y acción, la evaluación del desempeño y la interacción organizacional. Esta fase permitió a SERBILUZ, monitorear las actividades planificadas, detectar los puntos débiles y repotenciar las áreas que de acuerdo a los indicadores de gestión no respondían a los objetivos propuestos.

\section{Conclusiones}

La necesidad de incorporar en SERBILUZ un nuevo sistema de gestión basado en los principio de la NGP, surgió de un análisis estratégico básico, donde se consideraron los diferentes procesos desarrollados con el propósito de ofrecer servicios y productos informacionales a la comunidad intra y extrainstitucional. Por otra parte, hoy en día la nueva gerencia pública, que en su origen se concibió como un nexo entre el estado y la sociedad a través del mercado, ha tendido a 
centrarse en los problemas internos de la administración pública, por lo tanto sus criterios y lineamientos fueron perfectamente aplicables en SERBILUZ. Ello permitió la generación de las siguientes conclusiones:

Se auspició la conformación de un escenario favorable, para que el personal, tanto directivo como administrativo, alcanzara un nivel de motivación adecuado para lograr la excelencia en su desempeño laboral.

Como un signo altamente positivo, ha comenzado a surgir una cultura organizacional basada en la NGP, donde se ha declarado el reto de consolidar una gerencia sustentada en el modelo de las Cinco "R" propuesto por Osborne y Gaebler que favorecerá enfrentar las exigencias de los nuevos paradigmas informacionales.

La aplicación de este modelo fortaleció: el trabajo en equipo, los principios de aprender haciendo y aprender a aprender, la integración y aplanamiento de la estructura, a través de la reducción de los niveles jerárquicos, el mejoramiento de la comunicación interna y el establecimiento de políticas de estímulo e indicadores de desempeño y el establecimiento de alianzas estratégicas en el ámbito nacional e internacional que propiciaron el desarrollo eficiente de la info y tecnoestructura.

Es importante destacar que el entrenamiento, actualización y desarrollo dirigidos hacia el personal ubicado en todos los niveles de la organización fue el hilo conductor del proceso de transformación gerencial en SERBILUZ. En este sentido, se recomienda mantener el programa de adiestramiento y educación continúa de todo el personal que conforma el colectivo laboral, con la finalidad de profundizar los valores y principios de la cultura organizacional de SERBILUZ y proyectarlos permanentemente hacia un desempeño laboral de excelencia, además de perfeccionar e innovar los productos y servicios destinados a los usuarios.

\section{Referencias Bibliográficas}

Álvarez Trillos, José A. (2004). El desarrollo sostenible: gerenciando el futuro. En www.tablero-decomando.com (16-05-2004).

Ávalos Aguilar, Roberto (2002). Innovación de la Gestión Pública: Análisis y Perpectiva. En Edición Internet Gestión y Estrategia.

Dirección General de Planificación Universitaria (1998). Plan de Desarrollo Estratégico 1996-2000. Maracaibo. Universidad del Zulia.

Fernández, Norcka; Ortega, Egla; Delgado, Francys y Caldera, Edixson (2004). Logros por proyectos Estratégicos. Enero 2003-Abril 2004. Maracaibo: Comisión Reestructuradora de SERBILUZ. Universidad del Zulia.

Hammer, Michael y Champy, James C. (1997). Reingeniería. Colombia, Editorial Norma.

Hodd, Christopher (1994). Racionalismo económico en la gestión pública. De la administración pública progresiva a la nueva gestión pública. En Bruguet, Q. y Subirat, J. (1996). Lectura de gestión Pública. Madrid: Instituto Nacional de Administración Pública. Boletín Oficial del Estado. 468-469.

Hunter, James C. (1999). La Paradoja. Un relato sobre la verdadera esencia del Liderazgo. 5ta. ed. Barcelona, España: Ediciones Urano, S.A.

Jones, Lawrence R. y Thompson, Fred. (1997). "Un modelo para la nueva ge- 
rencia pública: lecciones de la reforma de los sectores públicos y privado". En Revista del CLAD № 15.

Koontz, Harold y Weihrich, Heinz (2004). Administración. Una Perspectiva Global. $12^{\mathrm{a}}$ ed. México: McGraw-Hill.

Medellín Torres, Pedro (1997). "Inestabilidad, Incertidumbre y Autonomía restringida: Elementos para una teoría de la estructuración de políticas publicas en países de baja autonomía gubernativa. En: Reforma y Democracia. Revista del CLAD. No. 8 pp.39-99.

Méndez, José Luis (1999). "Estudio Introductorio”. En Peter Guy B. La Política de la Burocracia. México: Colegio Nacional de Ciencias Políticas y Administración Pública, A.C. Fondo de Cultura Económica. Pp. 7-58.

Ortega, Egla y Ferrer, Jesús (2004). Gestión de la Información y el Conocimiento. Enero 2000-Abril 2004. Maracaibo: Comisión Reestructuradora de SERBILUZ. Universidad del Zulia.

Ortega, Egla y Pasternina, Joselyn (2003). TaIler Plan Operativo. Maracaibo: Comisión Reestructuradora de SERBILUZ. Universidad del Zulia.

Osborne, David y Gaebler, Ted (1999). La reinvención del gobierno: la influencia del espíritu empresarial en el sector público. Ediciones Paidos. Estado y Sociedad, 22.
Pérez Salgado, Ignacio (2000). "La inversión en recursos humanos de excelencia y la nueva gerencia pública". En Revista Centroamericana de Administración Pública. No. 38-39/Enero-Dic. Pp. 71-89.

Regos Varela, Xose (1999). "Programa de gestión y desarrollo de colecciones en una biblioteca universitaria (II). Políticas de gestión de colecciones". En: Boletín de la Asociación Andaluza de Bibliotecarios № 58. http://www. aab.es/Baab58a3.html (10-05-2004).

Shepherd, Goffrey (1999). El desafío de la reforma administrativa en América Latina. Revista del CLAD: Reforma y Democracia. Caracas. No. 13. Pp. 103-108.

Quintín, Antonio (2002). Paradigmas emergentes de la reforma administrativa: la nueva gestión pública. www.consejolocal.org/Archivo1/paradigmas.pdf (10-05-2004)

UNESCO (1996). Los nuevos escenarios universitarios ante el fin de siglo. Memorias del Seminario Taller copatrocinado por la Asociación de Universidades. Grupo Montevideo. 